\title{
Säureanschläge in Kambodscha
}

\author{
Acid Attacks in Cambodia
}

Autor

Institut
C. Bendick

University of Health Sciences, Faculty of Medicine, Department of Dermatology, Phnom Penh, Kambodscha
Bibliografie

DOI http://dx.doi.org/ $10.1055 / \mathrm{s}-0030-1256197$

Online-Publikation: 24. 2. 2011

Akt Dermatol 2011; 37:

111-113 @ Georg Thieme

Verlag KG Stuttgart · New York ISSN 0340-2541

\section{Korrespondenzadresse}

\section{Dr. med. Christoph Bendick}

University of Health Sciences Faculty of Medicine, Department of Dermatology BP 1006

Phnom Penh

Kambodscha

cambodia_derma@yahoo.de

\section{Zusammenfassung \\ $\nabla$}

Wie auch in anderen süd- und südostasiatischen Ländern sind Anschläge mit hochprozentiger Säure in Kambodscha ein relativ häufig zu beobachtendes Verbrechen. Die Gründe hierfür sind vielfältig: Eifersucht, Ärger und Hass kommen ebenso in Betracht wie Auseinandersetzungen um Erbe oder Grundbesitz. Die resultierenden Verletzungen sind in der Regel sehr ernst und schwierig zu therapieren, insbesondere in einem Land mit so unterentwickelter medizinischer Infrastruktur wie dies in Kambodscha der Fall ist. Dies bedingt, dass Patienten oftmals unzurei-

Anschläge mit hochprozentiger Säure werden in Kambodscha - wie auch in anderen süd- und südostasiatischen Ländern - vergleichsweise häufig beobachtet. Meist handelt es sich um Rachebzw. Vergeltungsakte für ein tatsächlich oder vorgeblich erlittenes Unrecht.

Was ist technisch unter einem Säureanschlag zu verstehen? „Ein vorsätzlicher Angriff auf ein Individuum in Form des Werfens, Schüttens oder sonstigen Applizierens von Säure oder anderen korrosiven Substanzen mit dem Ziel, ernsthaft zu verletzen, zu entstellen, zu foltern oder zu töten“ [1]. Die Konsequenz einer derartigen Attacke ist für das Opfer in aller Regel katastrophal: Falls sie nicht zum Tode führt, verändert sie radikal die Perspektive eines Lebens, welches fortan oftmals „einer nie enden wollenden Folter“ [2] gleicht. Das Resultat eines Säureanschlages ist insofern besonders gravierend als sich die Säure in kürzester Zeit durch Haut, Muskeln, Knorpel und selbst Knochen hindurchfrisst, ein Vorgang, der mit qualvollen Schmerzen verbunden ist und der Überlebende in aller Regel physisch und psychisch schwer geschädigt sowie sozial isoliert und stigmatisiert hinterlässt. chend behandelt werden und über die Verätzung der Haut hinaus gravierende bleibende Behinderungen wie Erblindung oder Ertaubung entwickeln. Die psychologischen Folgeerscheinungen eines Säureanschlages manifestieren sich in Form lang anhaltender Angstattacken, Depressionen und Selbstmordtendenzen. Das Leid der meisten Opfer wird verstärkt durch eine unzureichende soziale und psychologische Begleitung im Gefolge des Anschlags sowie durch eine desinteressierte und korrupte Justiz, welche reiche und einflussreiche Täter auf Kosten der oft aus bildungsfernen und unterprivilegierten Schichten stammenden Opfer bevorzugt behandelt.

Die Konsequenzen eines Säureanschlags führen jedoch über das persönliche Schicksal eines Betroffenen hinaus und reichen ganz allgemein bis weit hinein in Fragen der medizinischen Versorgung, der sozialen Sicherung, des Strafrechts und schließlich der Mentalität einer Gesellschaft, in welcher solche Verbrechen begangen werden. Opfer sind in Kambodscha (im Unterschied zu anderen Ländern, in denen ganz vorwiegend Frauen betroffen sind) Männer und Frauen ungefähr zu gleichen Teilen. Etwa ein Drittel der Betroffenen hat mit der Intention des Anschlages jedoch gar nichts zu tun: Hier handelt es sich um Personen, die zufällig in der Nähe stehen, um Irrtümer auf Seiten des Täters oder - besonders tragisch - um Kinder auf dem Arm eines angegriffenen Erwachsenen.

Die Gründe einer derartigen Attacke können vielfältig sein. Während es sich in muslimischen Ländern sehr oft um die Vergeltung eines Mannes für tatsächliche oder angebliche Untreue seiner Frau, Tochter oder Schwester handelt, spielen in Kambodscha unterschiedliche Motive eine Rolle. Meist handelt es sich um innerfamiliäre Emotionen wie Hass, Ärger, Eifersucht oder Vergeltung, welche diese spezielle Form der häuslichen Ge- 


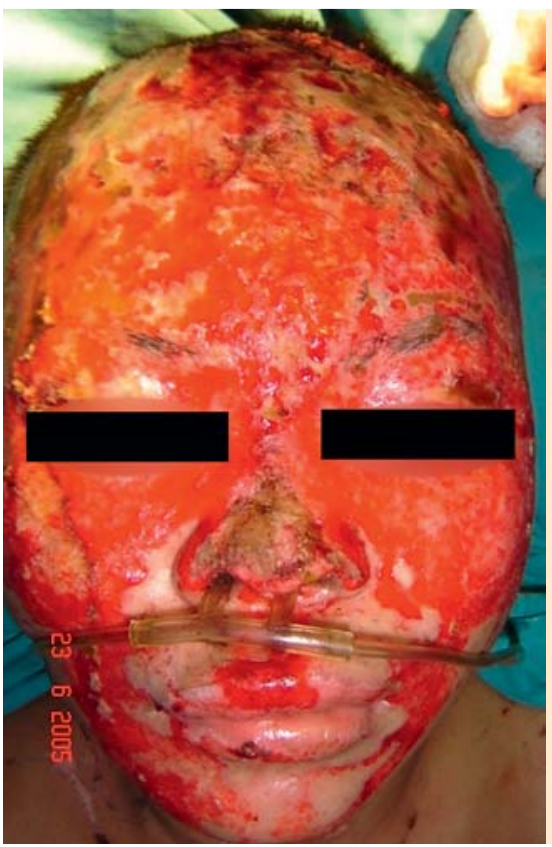

Abb. 1 Akute Säureverätzung, Z. n. Débridement $\left({ }^{\circ} \mathrm{Dr}\right.$ Jim Gollogly, Children's Surgical Center, Phnom Penh, Kambodscha).

walt bedingen. Aber auch geschäftliche Konflikte oder Streitigkeiten über Immobilien können Auslöser eines Anschlages sein [3]. In nicht wenigen Fällen bleibt die auslösende Ursache unklar, sei es, weil das Opfer nicht weiß, warum es angegriffen wurde, sei es, weil es nicht darüber sprechen möchte. Scham, Unsicherheit, Unbehagen im Umgang mit Polizei und Justiz sowie Angst vor einem Folgeanschlag lassen nicht wenige Betroffene davor zurückschrecken, auf Klärung zu drängen.

Hochprozentige Säure stellt in Kambodscha eine viel verwendete, leicht erhältliche und billige Substanz dar: Schwefelsäure wird für Autobatterien gebraucht, Salpetersäure wird von Gold- und Silberschmieden verwendet und Salzsäure ist ein notwendiger Stoff in der Latexproduktion [1]. Kaum verwunderlich ist es daher, dass die höchste Zahl von Säureanschlägen aus jenen Provinzen gemeldet wird, in denen Kautschuk verarbeitet wird. Säure kann in Kambodscha völlig legal und ohne jegliche Beschränkung gekauft, transportiert und verwendet werden. Gesetzliche Initiativen, den Erwerb hochprozentiger Säure zu reglementieren, wurden von Opferverbänden mehrfach angeregt, sind bislang jedoch im Dickicht der kambodschanischen Gesetzgebung stecken geblieben.

Der unmittelbare Effekt des Kontaktes mit Säure wird beschrieben als relativ unspezifisches Gefühl der Nässe und des Brennens auf der Haut. Bereits nach fünf Sekunden setzt eine Verätzung des oberflächlichen Gewebes ein; nach einer halben Minute ist die Haut unwiderruflich schwer geschädigt [4]. In aller Regel ist das Gesicht primäres Ziel der Attacke und hier kommt es zu den gravierendsten Einbußen: Augenlider, Ohren und Lippen schmelzen oftmals in kürzester Zeit bis zur Unkenntlichkeit, während Nasenlöcher, Gehörgänge und Atemwege verkleben. Die Haut von Rumpf und Extremitäten wird durch herunterlaufende und abtropfende Säure verätzt. Zügige Erste Hilfe kann die Schwere der Verletzungen deutlich reduzieren, aber Hilflosigkeit, Verwirrung und Angst vor dem Angreifer führen meist zu lähmender Untätigkeit auf Seiten der Zeugen eines Anschlages, während das Opfer selbst in seinem Schrecken kaum einen klaren und zielgerichteten Gedanken zu fassen vermag.

In aller Regel führen Attacken mit hochkonzentrierter Säure daher zu schwerwiegenden und bleibenden Behinderungen. Dabei kann es sich neben der erheblichen funktionell-ästhetischen Beein-

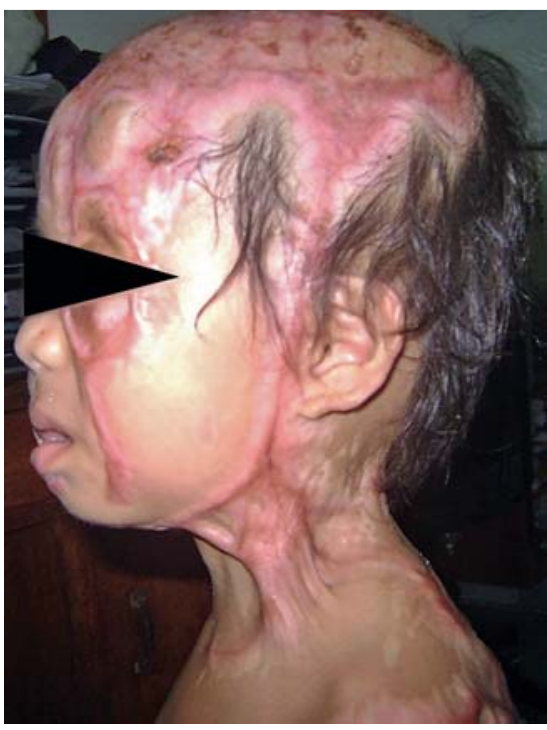

Abb. 2 Säureverätzung nach sechs Monaten: erheblicher Verlust des Kopfhaares, ausgeprägte Keloidstränge, Sklerosierung und Verziehung des linken Ober- und Unterlides $\left({ }^{\circ} \mathrm{Dr}\right.$ Jim Gollogly, Children's Surgical Center, Phnom Penh, Kambodscha).

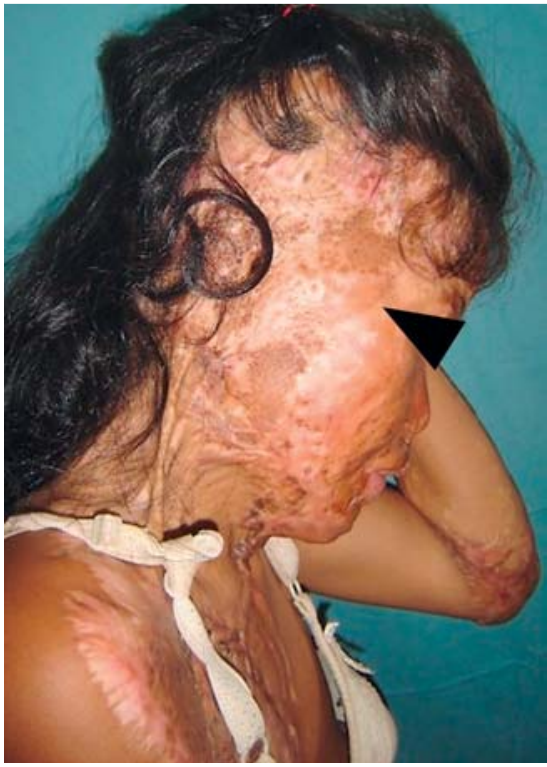

Abb. 3 Säureverätzung nach einem Jahr: partieller Verlust des Kopfhaares, Kontraktur des Ellenbogens, Keloidstränge, Poikilodermie ${ }^{\circ} \mathrm{Dr}$ Jim Gollogly, Children's Surgical Center, Phnom Penh, Kambodscha).

trächtigung durch chronische Ulzera, Narben, Keloide und Poikilodermie der Haut um die Einbuße oder schwere Schädigung des Seh- und Hörvermögens, den permanenten Verlust von Haaren, Augenbrauen und Wimpern sowie um Kontrakturen, insbesondere im Bereich der Arm- und Fingergelenke, handeln.

Erschwert wird die Lage in Kambodscha durch ein weithin unzureichend organisiertes, fachlich wenig kompetentes und technisch-apparativ völlig unterversorgtes Medizinwesen. Der im Rahmen einer Säureverätzung erforderliche rasche interdisziplinäre Ansatz von Verbrennungsspezialisten, Intensivmedizinern, plastischen Chirurgen, Ophthalmologen, Dermatologen und Psychologen stellt schon für ein Land mit entwickelter medizinischer Infrastruktur eine Herausforderung dar - in Kambodscha ist er derzeit noch nicht einmal ansatzweise vorhanden. Hinzu kommt die Notwendigkeit der sich an die Akutbehandlung anschließenden jahrelangen physischen und psychischen Rehabilitation, welche unter Drittwelt-Bedingungen nicht geleistet werden kann. Besonders dramatisch ist die Situation bei Kindern, deren Wachstum wesentlich höhere Anforderungen an die Quantität plastisch-operativer Maßnahmen stellt, als dies bei Erwachsenen der Fall ist [5]. Einige wenige Betroffene suchen Behandlung in umliegenden Ländern mit entwickelterer medizinischer Infrastruktur oder in 


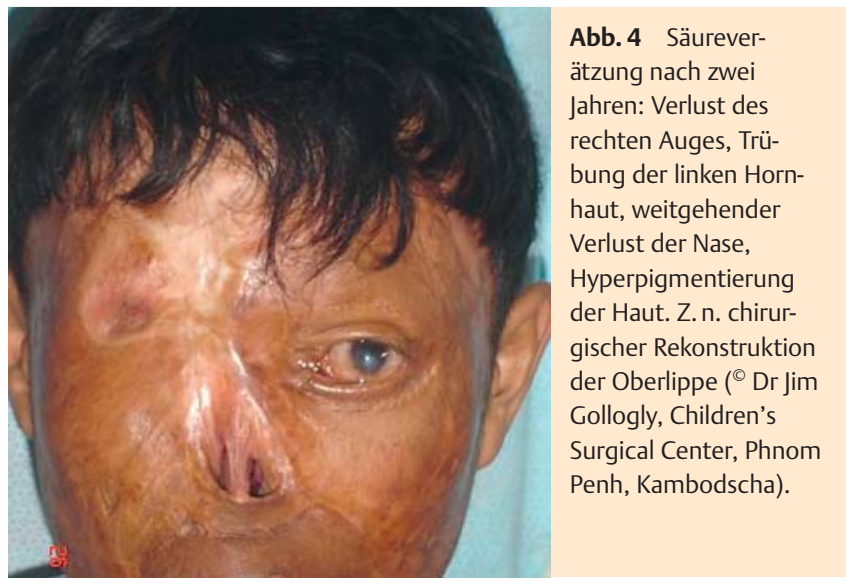

Einrichtungen der medizinischen Spitzenversorgung wie etwa in Singapur oder Australien, aber der übergroßen Mehrheit der Patienten bleiben diese Optionen aus finanziellen Gründen versagt. Über die körperliche Problematik hinaus sind Überlebende in der Regel mit erheblichen psychologischen, emotionalen and sozialen Folgen konfrontiert. Hierfür sind u.a. verantwortlich:

- der Anschlag selbst mit seiner traumatisierenden Erfahrung des Schmerzes, des Schreckens, der Angst und Hilflosigkeit,

- die Erkenntnis der kostspieligen Abhängigkeit von meist inadäquater ärztlicher Versorgung und daraus resultierender unzureichender körperlicher Besserung und finanzieller Verarmung,

- die Wahrnehmung, erotisch zerstört zu sein und vom Partner verlassen zu werden bzw. keinen Partner zu finden (wie in zahlreichen anderen asiatischen Ländern ist es in Kambodscha ein Zeichen von Minderwertigkeit, unverheiratet zu sein),

- durch körperliche Behinderung bzw. ästhetische Beeinträchtigung bedingte Stigmatisierung und der damit verbundene Ausschluss aus der Gemeinschaft,

- die Scham für den Umstand, ein wenig produktives Mitglied der Gesellschaft und somit auf die finanzielle Unterstützung anderer angewiesen zu sein.

Aus dieser massiven Belastung entstehen bei vielen Betroffenen quälende psychosomatische Symptome wie Kopfschmerzen, Herzrasen, Unruhe und Schlaflosigkeit. Die resultierenden Rückzugstendenzen können eine anhaltende depressive Verstimmung bis hin zur manifesten Depression und bis zum Suizid zur Folge haben.

Das bei den meisten Opfern vorherrschende Gefühl ist Angst: Angst vor der künftigen Lebenssituation, der Notwendigkeit anhaltender und kostspieliger medizinischer Behandlung, Angst vor dem Täter, vor weiteren Racheakten - kurz: Angst vor der Welt außerhalb des engsten Freundes- und Familienkreises. Nicht selten vertreten Außenstehende, insbesondere, wenn es sich bei der Geschädigten um eine Frau handelt, die Ansicht, dass der Anschlag schon einen Grund gehabt haben wird und dass das Opfer selbst nicht ganz unschuldig an dem ihm zugefügten Unrecht sein kann.

Diese Haltung ist mit einem in Kambodscha (übrigens nicht nur im Bereich von Säureanschlägen) weit verbreiteten Phänomen assoziiert: Straflosigkeit. Meist entgehen Täter der Anklage und Bestrafung, weil ein Anschlag mit Säure im öffentlichen Bewusstsein nicht wirklich als Kapitaldelikt wahrgenommen wird [3], es insofern dem Opfer überlassen ist, ob es strafrechtliche Verfolgung anstrebt oder nicht. Die vielfach aus bildungsfernen und finanziell schwachen Schichten stammenden Betroffenen verfü- gen meist jedoch nicht über die Kenntnisse, die Verbindungen und die finanziellen Mittel, um eine polizeiliche Anzeige, anwaltliche Vertretung und gerichtliche Aufarbeitung der Tat anzustreben. Hinzu kommt die Angst vor der Rache des Verursachers. Täter erreichen es nicht selten, eine strafrechtliche Verfolgung durch Drohungen an die Adresse des Betroffenen und Bestechung von Polizei und Justiz zu blockieren. Insbesondere reiche und politisch oder gesellschaftlich einflussreiche Täter werden in aller Regel von einer weithin unterqualifizierten, desinteressierten und beeinflussbaren Justiz noch nicht einmal ansatzweise belangt [6]. In Fällen, in denen es doch zur Anklage kommt, werden Täter meist mit völlig inadäquaten Geld- oder Haftstrafen belegt. Insofern ist ein Vertrauen der Betroffenen in die zuständigen staatlichen Organe kaum gegeben.

Auf wiederholtes Drängen von Nichtregierungs-Organisationen, Menschenrechtsgruppen und Opferverbänden wurde von den verantwortlichen Behörden nachdrücklich eine verbesserte Gesetzgebung zur konsequenteren Verfolgung von Säureattentätern gefordert, entsprechende Initiativen scheinen (im Gegensatz etwa zu Bangladesch) in Kambodscha jedoch bislang keine vorrangige Priorität zu haben.

\section{Abstract}

\section{Acid Attacks in Cambodia}

\section{$\nabla$}

As in other South- and Southeast-Asian countries, attacks with highly concentrated acid constitute a rather frequent crime in Cambodia. Reasons for this are varied: jealousy, anger, hatred as well as altercations about property can play a role. Ensuing injuries are usually grave and difficult to treat, in particular in a country with a poor medical infrastructure as is the case in Cambodia. Thus, patients are often insufficiently treated and suffer therefore from permanent physical disabilities like blindness or deafness on top of their aesthetic impairment. The psychological aftermath of an acid-attack is characterized by anxiety, depression and suicidal ideations. The overall helplessness of victims is enhanced by poor social services to cope with the consequences of the attack as well as a disinterested and corrupt judiciary which favours rich and influential perpetrators over deprived victims.

\section{Literatur}

1 Gollogly JG, Say Bon Vath, Malmberg A. Acid Attacks in Cambodia: Serious Reminders of Personal Conflicts. Asian Biomedicine 2008; 2: $329-334$

2 LICADHO. Living in the Shadows: Acid Attacks in Cambodia, 2003. Eingesehen online unter: www.licadho-cambodia.org/reports.php? perm=41; Stand: 15.10 .2010

3 Cambodian Acid Survivors Charity (CASC). Breaking the Silence: Addressing Acid Attacks in Cambodia. May 2010

Eingesehen online unter: www.sithi.org/admin/upload/media/[201005-24]1230/Breaking\%20the\%20Silence\%20-\%20Addressing\%20Acid\% 20Attacks\%20in\%20Cambodia.pdf; Stand: 20.10.2010

4 Shahidul BM, Iqbal Mahmud CM. Acid Burns in Bangladesh. Annals of Burns and Fire Disasters; Vol. 14, No. 3, September 2001

5 ASTI. Where It Happens. Cambodia.

Eingesehen online unter: www.acidviolence.org/index.php/acid-violence/countries/cambodia; Stand: 24.10.2010

6 Siehe hierzu, auf Bangladesch bezogen, auch: Haque M. Impunity and Acid Violence Against Women in Bangladesh: Case Studies in Satkhira and Sirajgonj Districts. M.A. Thesis University of Mahidol: 2005 Eingesehen online unter: http://mulinet10.li.mahidol.ac.th/e-thesis/ 4737344.pdf; Stand: 22.10.2010 